\title{
Preventing stroke after transient ischemic attack
}

\author{
Michael D. Hill MD MSc, Shelagh B. Coutts MD
}

See related research article by Perry and colleagues on page 1137 and at www.cmaj.ca/lookup/doi/10.1503/cmaj.101668.

$\mathrm{S}$ troke is a syndrome that can have either an ischemic or hemorrhagic cause. It is now clear that transient ischemic attack and minor ischemic stroke are highly predictive of a subsequent disabling ischemic stroke within hours or days. The critical clinical problems for physicians and nurse practitioners who evaluate these patients are to identify that the patient has had a stroke or transient ischemic attack and to stratify risk to determine if the patient requires rapid intervention to prevent recurrent stroke.

Diagnosing transient ischemic attack and minor ischemic stroke is not easy. Transient ischemic attacks are sudden. Although maximal symptoms occur at onset, it is a historical diagnosis since patients usually have no clinical signs by the time they are evaluated. Most transient ischemic attacks last 15 minutes or less. ${ }^{1}$ Persistent symptoms or signs, however minor, imply a diagnosis of minor stroke. However, this technical distinction is rarely made by clinicians in routine practice or in the current stroke literature.

Common mimics of minor stroke or transient ischemic attack include migrainous aura, simple partial seizures and somatization. Stroke is the most common sudden neurologic event affecting adults; ischemia should be the default diagnosis when there is uncertainty. Symptoms are related to the area of the brain that is dysfunctional rather than to the cause of the dysfunction, making diagnostic inference difficult. Imaging is helpful, particularly diffusion-weighted magnetic resonance imaging, in which a positive result rules in the diagnosis of ischemia. A fast magnetic resonance protocol for stroke consisting of an axial diffusionweighted image, axial fluid-attenuated inversion recovery imaging and axial gradient-recalled echo imaging (to detect hemorrhage) takes less than 10 minutes of imaging time and could be made available in all large hospitals in Canada. A normal magnetic resonance image does not rule out a diagnosis of ischemia, but provides confidence that the immediate risk of stroke is low., ${ }^{2,3}$

Diagnosing transient ischemic attack identifies a patient who is at risk for subsequent stroke. The risk of stroke after transient ischemic attack is somewhere between $2 \%$ and $17 \%$ within the first 90 days. Among patients with transient ischemic attack, one in five will have a subsequent stroke (the most common outcome), a heart attack or die within one year. ${ }^{4,5}$ Recurrent stroke can occur in one of two ways: ${ }^{1}$ the initial stroke or transient ischemic attack progresses and worsens, ${ }^{2}$ with ischemia evolving in the initial vascular territory; or a subsequent ischemic event occurs (e.g., a new embolic event) in the same or new vascular territory. Each situation depends on the mechanism of the initial event and the residual vascular patency.

A high proportion of strokes are due to embolic (arteroembolic or cardioembolic) occlusion of intracranial arteries. Early assessment of intracranial and extracranial vessels is critical to assessing risk. Patients with persistent intracranial occlusions have a four-fold increase in risk of developing recurrent stroke. ${ }^{6}$ Stroke caused by arteroemboli from large atherosclerotic arteries (e.g., extracranial carotid or vertebral artery) can recur early after the initial event. These causes can be quickly identified with imaging of the intracranial and extracranial arteries. Lacunar syndromes are more challenging because the small penetrating arteries are too small to see using current imaging technology.

Cardioembolic stroke events tend not to recur early, and there is no clear evidence that anticoagulation prevents recurrence within the first two weeks post-event; therefore, the cardiac work up can be done as an outpatient.

Understanding the mechanism and vascular diagnosis involved in transient ischemic attack and stroke allows a rational approach to early intervention. This is not afforded by the ABCD2 score (age, blood pressure, clinical features, duration of symptoms and diabetes), which relies on clinical risk factors, and explains why the $\mathrm{ABCD} 2$ is largely an ineffective screening tool.

\section{KEY POINTS}

- Transient ischemic attack and minor stroke are highly predictive of a subsequent disabling stroke within hours or days of the first event.

- The risk of subsequent stroke after a transient ischemic attack is between $2 \%$ and $17 \%$ within the first 90 days after the initial event.

- Understanding the mechanism of the stroke syndrome allows a rational approach to early intervention.
Competing interests:

Shelagh Coutts has received a salary award from the Heart and Stroke Foundation of

Canada and Alberta Innovates Health Solutions, and grants from the Canadian Institutes of Health Research, the Heart and Stroke

Foundation of Canada, the Canadian Stroke Network and Pfizer. Michael Hill has received support from the

Canadian Institutes of Health Research, the United States National Institutes of Health, Alberta Innovates Health

Solutions, Hoffman-

La Roche Canada, Bayer

Canada and Merck Canada;

he is a member of the board of the Heart and Stroke

Foundation of Alberta, NWT \& Nunavut, from whom he has received a professorship; he has been a consultant for the Vernalis Group Ltd., Stem Cell Therapeutics and Portola Therapeutics; he has received speaker fees from Sanofi-Aventis Canada, Bristol-Myers Squibb and Boehringer Ingelheim Canada; he holds stock in Calgary Scientific Inc., and he has been reimbursed for travel expenses by GE Canada Healthcare.

This article was solicited and has not been peer reviewed.

Correspondence to:

Dr. Michael Hill, hillmd@ucalgary.ca

CMAJ 2011. DOI:10.1503 /cmaj.110704 
The ABCD2 score was never designed to be used as a screening tool. Indeed, the initial studies used to develop and validate the ABCD2 score showed that it had only moderate discriminative value. ${ }^{7}$ In this issue of the CMAJ, Perry and colleagues show that the score has insufficient sensitivity and specificity to be of use in the urgent evaluation of patients. ${ }^{8}$

It is possible that the failure to show a reasonable discriminative value for the ABCD2 score is due to improvements in stroke care; ${ }^{9,10}$ transient ischemic attack and minor stroke are being treated more aggressively. The ABCD2 score was developed using data from populations that did not receive hyperacute stroke prevention. Because outcomes have improved with early treatment, any tool for identifying risk will need to adapt to the changing prevalence of poor outcomes.

The ABCD2 score has raised awareness about the importance of identifying transient ischemic attack and minor stroke as a medical urgency requiring same-day assessment and management. The most important prognostic component of the $\mathrm{ABCD} 2$ score is the " $\mathrm{C}$ " for clinical symptoms. ${ }^{11}$ Motor and speech symptoms, when present, increase the probability that the patient has truly had brain ischemia. Isolated sensory events are more likely to be due to alternate causes of acute symptoms, such as migrainous aura, a simple partial seizure or somatization. ${ }^{12}$

The approach to caring for the patient with acute, sudden, neurologic symptoms that have resolved is first to make the correct diagnosis and then to stratify risk so that appropriate decisions regarding triage and treatment can be made.

Imaging of the brain and extracranial and intracranial blood vessels (aortic arch to vertex) can be done with magnetic resonance angiography, but higher resolution imaging is available with computed tomography angiographs. ${ }^{13}$ Persistent arterial occlusions (partial or complete), protruding thrombus in the artery, lacunar syndromes, significant carotid artery disease, all appropriate to the predicted relevant ischemic region of the brain, warrant admission to a hospital or clinic for same-day urgent assessment.

Treatment follows diagnosis. All patients should receive a loading dose of antiplatelet medication. Low-dose acetylsalicylic acid is the usual course of treatment. Patients with new onset of atrial fibrillation without a persisting vascular occlusion can be given treatment with anticoagulant medications and sent home. In Calgary, our approach to treatment is based on the mechanism of the event and vascular patency, but there are many patients for whom treatment remains gen eric because there is no specific vascular diagnosis, the diagnosis of transient ischemic attack is uncertain, or the immediate diagnostic work-up is inadequate. There is a great need for therapeutic trials in this area. The FASTER trial ${ }^{14}$ suggested that double-antiplatelet therapy might be helpful, and the POINT trial (clinical trial number NCT00991029) may definitively answer whether such an approach is useful. However, several questions remain. For example, we do not know how to manage blood pressure acutely or how quickly carotid intervention should be done.

Stroke management is becoming more complex and clinical rules are no longer sufficient. The diagnosis of a stroke syndrome, once made, implies the need to investigate the vascular mechanism that caused it. The ABCD2 score has raised awareness. Imaging has elevated understanding. Therapeutic trials are next.

\section{References}

1. Levy DE. How transient are transient ischemic attacks? Neurology 1988;38:674-7.

2. Coutts SB, Eliasziw M, Hill MD, et al. An improved scoring system for identifying patients at high early risk of stroke and functional impairment after an acute transient ischemic attack or minor stroke. Int J Stroke 2008;3:3-10.

3. Coutts SB, Simon JE, Eliasziw M, et al. Triaging transient ischemic attack and minor stroke patients using acute magnetic resonance imaging. Ann Neurol 2005;57:848-54.

4. Wu CM, McLaughlin K, Lorenzetti DL, et al. Early risk of stroke after transient ischemic attack: a systematic review and meta-analysis. Arch Intern Med 2007;167:2417-22.

5. Hill MD, Yiannakoulias N, Jeerakathil T, et al. The high risk of stroke immediately after transient ischemic attack: a populationbased study. Neurology 2004;62:2015-20.

6. Coutts SB, Hill MD, Demchuk AM, et al. Early CT/CTA predict recurrent events in minor stroke/TIA patients: main results of the CT and MRI in the triage of TIA and minor cerebrovascular events to identify high risk patients $(\mathrm{CATCH})$ study. Cerebrovasc Dis 2011;31(Suppl. 2):68

7. Rothwell PM, Giles MF, Flossmann E, et al. A simple score $(\mathrm{ABCD})$ to identify individuals at high early risk of stroke after transient ischaemic attack. Lancet 2005;366:29-36.

8. Perry JJ, Sharma M, Silvotti M, et al. Prospective validation of the $\mathrm{ABCD} 2$ score for patients in the emergency department with transient ischemic attack. CMAJ 2011;183:1137-45.

9. Lavallée PC, Meseguer E, Abboud H, et al. A transient ischaemic attack clinic with round-the-clock access (SOS-TIA): feasibility and effects. Lancet Neurol 2007;6:953-60.

10. Rothwell PM, Giles MF, Chandratheva A, et al. Effect of urgent treatment of transient ischaemic attack and minor stroke on early recurrent stroke (EXPRESS study): a prospective populationbased sequential comparison. Lancet 2007;370:1432-42.

11. Coutts SB, Sylaja PN, Choi YB, et al. The ASPIRE approach for TIA risk stratification. Can J Neurol Sci 2011;38:78-81.

12. Johnston SC, Sidney S, Bernstein AL, et al. A comparison of risk factors for recurrent TIA and stroke in patients diagnosed with TIA. Neurology 2003;60:280-5.

13. Coutts SB, O'Reilly C, Hill MD, et al. Computed tomography and computed tomography angiography findings predict functional impairment in patients with minor stroke and transient ischaemic attack. Int J Stroke 2009;4:448-53.

14. Kennedy J, Hill MD, Ryckborst KJ, et al. Fast assessment of stroke and transient ischaemic attack to prevent early recurrence (FASTER): a randomised controlled pilot trial. Lancet Neurol 2007;6:961-9.

Affiliations: From the Department of Clinical Neurosciences, Hotchkiss Brain Institute, University of Calgary, Foothills Medical Centre, Calgary, Alta.

Contributors: Michael D. Hill wrote the initial draft of the manuscript. Both authors contributed to the writing and revision of the manuscript and approved the final version submitted for publication. 